Lucas Henrique Rosso ${ }^{1}$

Dhttps://orcid org/0000-0001-9217-0995

Guilherme Assoni Gomes'

(- https://orcid.org/0000-0003-2998-0877

Luis Felipe Chaga Maronezi ${ }^{1}$

@isttps://orcid.org/0000-0001-7947-939X

Ivana Loraine Lindemann

(-) https://orcid.org/0000-0002-6222-9746

Rogério Tomasi Riffel’

Ohttps://orcid.org/0000-0002-2285-204X

Júlio César Stobbe

Ohttps://orcid.org0000-0001-9329-2919

\section{Delirium em idosos internados via unidades de emergência: um estudo prospectivo}

Delirium in elderly inpatients in emergency units: a prospective study

DOI: 10.1590/0047-2085000000261

\section{RESUMO}

Objetivos: Determinar a incidência de delirium em idosos internados em um hospital terciário e verificar sua distribuição de acordo com variáveis preditoras. Métodos: Foram incluídos idosos que se internaram via emergência de abril a setembro de 2018. A amostra foi caracterizada e foi verificada a diferença da incidência de delirium, em até cinco dias de internação, em relação às variáveis preditoras (teste qui-quadrado, 5\%). Resultados: A amostra foi composta por 300 participantes predominantemente do sexo masculino (52\%), com idade entre 60 e 69 anos (50,7\%), eutróficos (49,3\%) e com três ou mais doenças anteriores à internação (42\%). A incidência de delirium foi de 12\% (IC95\% 9-16). Foi observada diferença estatisticamente significativa em relação ao número de doenças anteriores à internação ( $p=0,025)$, à saída do leito durante a internação $(p<0,001)$ e ao uso de sonda vesical $(p<$ 0,001). Conclusão: $O$ reconhecimento desse transtorno é fundamental na rotina de emergência dos hospitais, onde são atendidos pacientes com múltiplas doenças crônicas, pois o delirium é comumente subdiagnosticado, levando a um aumento nas taxas de morbimortalidade.

\section{PALAVRAS-CHAVE}

Transtornos neurocognitivos, idoso, serviços médicos de emergência.

\section{ABSTRACT}

Objectives: To determine the incidence of delirium in the elderly admitted to a tertiary hospital and to verify its distribution according to predictor variables. Methods: Elderly patients admitted to the emergency room from April to September 2018 were included. The sample was characterized and the difference in the incidence of delirium within five days if hospitalization regarding the predictor variables was observed (Chi-square test, 5\%). Results: The sample consisted of 300 predominantly male participants (52\%), aged 60-69 years (50.7\%), eutrophic (49.3\%) and with three or more pre-hospitalization diseases (42\%). The incidence of delirium was 12\% (95\% Cl 9-16). A statistically significant difference was observed in relation to the number of diseases prior to hospitalization $(p=0.025)$, the exit from bed during hospitalization $(p<0.001)$ and the use of bladder tube $(p<0.001)$. Conclusion: The recognition of this disorder is fundamental in the emergency routine of hospitals, where patients with multiple chronic diseases are treated, as delirium commonly underdiagnosed, leading to an increase in morbidity and mortality rates.

KEYWORDS

Neurocognitive disorders, aged, emergency medical services. 


\section{INTRODUÇÃO}

O delirium, também chamado de estado confusional agudo, configura-se como um distúrbio neuropsiquiátrico muito comum em ambiente hospitalar, particularmente em idosos'. A epidemiologia é bastante diversificada, sobretudo pela variação da idade, situação clínica e contexto de intervenção no paciente. Um terço dos pacientes com mais de 70 anos apresenta o distúrbio na admissão hospitalar e outra metade, durante o período de internação². Também é um quadro frequente naqueles submetidos à cirurgia, com risco entre $10 \%$ e $50 \%$, principalmente em fraturas de quadril e intervenções cardíacas ${ }^{3}$. Já em unidade de terapia intensiva (UTI) a frequência pode atingir $80 \%{ }^{4}$.

Está associado a maior tempo de internação, alto custo nos serviços de saúde, dano funcional e cognitivo em longo prazo e aumento da mortalidade. Estima-se que nos EUA o estado confusional agudo seja responsável por um acréscimo de 60 mil dólares por paciente/ano, o que resulta em sobrecarga econômica de 38 a 152 bilhões de dólares anualmente ${ }^{5}$. Os dados brasileiros são escassos a respeito da epidemiologia, entretanto a doença foi responsável por 56\% das interconsultas psiquiátricas em idosos em um hospital geral ${ }^{6}$.

De acordo com o Manual Diagnóstico e Estatístico de Transtornos Mentais, quinta edição (DSM-5) ${ }^{7}$, o delirium requer uma perturbação na atenção e na consciência, que se desenvolve agudamente e tende a flutuar. Isso resulta em um distúrbio cognitivo ou perceptivo que não é mais bem explicado por um quadro de demência preexistente, estabelecida ou em evolução.

Constitui-se em uma síndrome multifatorial e vários fatores exercem efeitos favoráveis a essa desordem neuropsíquica. Dentre eles, destacam-se idade avançada, sexo masculino, déficit sensorial, história de alcoolismo, depressão e doenças neurológicas. Além disso, podem existir fatores precipitantes ao quadro de delirium como doença aguda grave, polifarmácia, desidratação, distúrbios metabólicos, desnutrição, entre outros ${ }^{8}$.

Diante desse panorama, o objetivo deste estudo foi determinar a incidência de delirium em idosos internados em um hospital terciário, bem como verificar sua distribuição de acordo com variáveis preditoras.

\section{MÉTODOS}

Trata-se de um estudo clínico, observacional e prospectivo, realizado em um hospital terciário de ensino, sendo a amostra constituída por pacientes de ambos os sexos, com idade igual ou superior a 60 anos, que se internaram via emergência entre abril e setembro de 2018. Foram excluídos aqueles que estavam desorientados e não apresentavam condições de responder aos instrumentos de coleta de dados, bem como aqueles com antecedentes de acidente vascular encefálico, demência ou outra doença cerebrovascular, politraumatizados, em estado cardiorrespiratório instável e internados para cirurgia eletiva.

A coleta de dados foi realizada em três momentos distintos. Primeira: diariamente, entre as $06 \mathrm{~h} 00$ e as $12 \mathrm{~h} 00$ e entre as 18 h00 e a 00h00, na secretaria do setor de emergência, era obtida a lista dos pacientes internados no dia e feita a consulta ao sistema de informações hospitalares para a verificação dos critérios de seleção. A partir disso, os pacientes selecionados eram convidados a participar do estudo e, em caso de consentimento, era aplicado o Miniexame do Estado Mental (MEEM) ${ }^{9}$, instrumento fácil e rápido, utilizado para avaliar a função cognitiva, com pontos de corte estabelecidos de acordo com o nível de escolaridade do indivíduo. Em seguida, o algoritmo Confusional Assessment Method (CAM), instrumento capaz de ser aplicado em menos de 5 minutos, que avalia a presença do estado confusional agudo, composto por nove critérios clínicos, dos quais os quatro primeiros mostram maior sensibilidade e especificidade para a detecção do quadro: início agudo e curso flutuante, desatenção, pensamento desorganizado e alteração do nível de consciência ${ }^{10}$.

Foram considerados positivos para delirium e excluídos do estudo os pacientes que apresentavam os critérios 1 e 2, mais os critérios 3 e/ou 4 da referida escala. Nos pacientes negativos para delirium, a coleta de dados prosseguia, com aplicação de questionário e busca em prontuário: sexo, idade, cor da pele, renda mensal individual, local de residência, condições de moradia, tabagismo, consumo de bebida alcoólica, prática regular de atividade física, peso, altura, número de doenças anteriores à internação, doença cardiovascular, infecciosa e/ou psiquiátrica, tanto prévias quanto apresentadas durante a permanência no hospital, saída do leito durante a internação, uso de sonda nasogástrica, uso de sonda vesical de demora, uso de cateter venoso central, realização de fisioterapia, cirurgia na internação atual, uso de opioides, uso de hipnóticos/sedativos e uso de anti-hipertensivos, prévios ou iniciados na internação.

Segunda coleta: no terceiro dia da internação (72 horas), foi reaplicado o algoritmo CAM (ainda no setor de emergência ou nas enfermarias, conforme a disponibilidade de leitos no hospital) e buscaram-se novos dados em prontuário: introdução de sondagem nasogástrica, vesical e/ou de cateter de acesso venoso central, saída do leito, diagnóstico de doença psiquiátrica, cardiovascular ou infecciosa, início de fisioterapia, introdução de opioide, hipnótico/sedativo ou anti-hipertensivo e realização de procedimentos cirúrgicos. Em caso de delirium, a coleta era interrompida.

Terceira coleta: nos pacientes negativos para delirium no terceiro dia, no quinto dia de internação (120 horas), ainda 
no setor de emergência ou nas enfermarias, conforme a disponibilidade de leitos no hospital, repetiu-se a coleta realizada no terceiro dia.

Da segunda e da terceira etapa, foram excluídos os pacientes que evoluíram a óbito e os que obtiveram alta hospitalar ou que foram transferidos para UTI.

Após digitação e validação dos dados, foi considerada como variável dependente a incidência de delirium em até cinco dias de internação (incidência no terceiro e no quinto dia), sendo analisadas como preditoras as variáveis sexo, idade, consumo de bebida alcoólica, resultado do MEEM, número de doenças anteriores à internação, doença cardiovascular, infecciosa e psiquiátrica, deambulação, uso de sonda vesical, nasogástrica e de cateter venoso central, fisioterapia, cirurgias e uso de opioides, de hipnóticos/sedativos e de anti-hipertensivos. Procedeu-se à estatística descritiva, ao cálculo da incidência do delirium (IC de 95\%) e à verificação da sua distribuição de acordo com as variáveis preditoras (teste do qui-quadrado, 5\%).

O protocolo de estudo foi aprovado pelo Comitê de Ética em Pesquisa com Seres Humanos da instituição proponente, em conformidade com a Resolução no 466/2012 do Conselho Nacional de Saúde.

\section{RESULTADOS}

A amostra foi composta por 300 idosos caracterizados predominantemente como do sexo masculino (52\%), com idade entre 60 e 69 anos (50,7\%), cor da pele branca (73,7\%), renda mensal entre 1 e 2 salários mínimos (51,6\%), residentes em zona urbana $(78,6 \%)$, em casa ou apartamento com parentes ou outras pessoas (49\%). No que se refere aos hábitos de vida, 44\% não eram tabagistas, 85,8\% não consumiam bebida alcoólica e 65,9\% não praticavam atividade física.

Em relação à caracterização da saúde, a maior parte dos idosos obteve a pontuação mínima no MEEM $(54,7 \%)$, era eutrófica (49,3\%) e apresentava três ou mais doenças prévias à internação (42\%), sendo 80\% doença cardiovascular, 9\% infecciosa e 8,3\% psiquiátrica. A maioria deambulou durante a internação $(76,4 \%)$ e $17,7 \%$ fizeram uso de sonda vesical, $8,2 \%$ de sonda nasogástrica e 5,5\% de cateter venoso central. Fisioterapia foi realizada por 39,6\% e 20,3\% foram submetidos a cirurgia no período. Em relação aos medicamentos, 29,4\% usaram opioide, 49,6\%, hipnótico/sedativo e 85,7\%, anti-hipertensivos (Tabela 1).

A incidência de delirium foi de 12\% (IC95\% 9-16) e a tabela 2 apresenta a sua distribuição de acordo com as variáveis preditoras. Foi observada diferença estatisticamente significativa em relação ao número de doenças anteriores à internação $(p=0,025)$, à saída do leito durante a internação ( $p<$ $0,001)$ e ao uso de sonda vesical $(p<0,001)$.
Tabela 1. Caracterização sociodemográfica, de hábitos de vida e de saúde de uma amostra de idosos internados via emergência em um hospital terciário - Passo Fundo, RS, $2018(n=300)$

\begin{tabular}{|c|c|c|}
\hline Variáveis & $\mathbf{n}$ & $\%$ \\
\hline \multicolumn{3}{|l|}{ Sexo } \\
\hline Masculino & 156 & 52,0 \\
\hline Feminino & 144 & 48,0 \\
\hline \multicolumn{3}{|l|}{ Idade (anos completos) } \\
\hline $60-69$ & 152 & 50,7 \\
\hline $70-79$ & 90 & 30,0 \\
\hline$\geq 80$ & 58 & 19,3 \\
\hline \multicolumn{3}{|l|}{ Cor da pele } \\
\hline Branca & 221 & 73,7 \\
\hline Outras & 79 & 26,3 \\
\hline \multicolumn{3}{|l|}{ Renda mensal em salários mínimos $(n=279)^{\star}$} \\
\hline$\leq 1$ & 90 & 32,3 \\
\hline $1-2$ & 144 & 51,6 \\
\hline$>2$ & 45 & 16,1 \\
\hline \multicolumn{3}{|l|}{ Local de residência ( $n=294)$} \\
\hline Zona urbana & 231 & 78,6 \\
\hline Zona rural & 63 & 21,4 \\
\hline \multicolumn{3}{|l|}{ Condições de residência ( $n=298)$} \\
\hline Casa ou apartamento sozinho & 27 & 9,1 \\
\hline Casa ou apartamento com cônjuge & 125 & 41,9 \\
\hline Casa ou apartamento com parentes ou outras pessoas & 146 & 49,0 \\
\hline \multicolumn{3}{|l|}{ Tabagismo $(n=298)$} \\
\hline $\operatorname{Sim}$ & 43 & 14,4 \\
\hline Não & 131 & 44,0 \\
\hline Ex-tabagista & 124 & 41,6 \\
\hline \multicolumn{3}{|l|}{ Consumo de bebida alcoólica ( $n=296)$} \\
\hline $\operatorname{Sim}$ & 42 & 14,2 \\
\hline Não & 254 & 85,8 \\
\hline \multicolumn{3}{|l|}{ Atividade física $(n=293)$} \\
\hline $\operatorname{Sim}$ & 100 & 34,1 \\
\hline Não & 193 & 65,9 \\
\hline \multicolumn{3}{|l|}{ Miniexame do Estado Mental } \\
\hline Aprovado & 164 & 54,7 \\
\hline Não aprovado & 136 & 45,3 \\
\hline \multicolumn{3}{|l|}{ Estado nutricional $(n=280)$} \\
\hline Baixo peso & 36 & 12,9 \\
\hline Eutrofia & 138 & 49,3 \\
\hline Sobrepeso & 106 & 37,8 \\
\hline \multicolumn{3}{|l|}{ Número de doenças anteriores à internação } \\
\hline $0-1$ & 90 & 30,0 \\
\hline 2 & 84 & 28,0 \\
\hline$\geq 3$ & 126 & 42,0 \\
\hline Doença cardiovascular & 240 & 80,0 \\
\hline Doença infecciosa & 27 & 9,0 \\
\hline Doença psiquiátrica & 25 & 8,3 \\
\hline Deambulação ( $n=144)$ & 110 & 76,4 \\
\hline Uso de sonda vesical $(n=147)$ & 26 & 17,7 \\
\hline Uso de sonda nasogástrica $(n=146)$ & 12 & 8,2 \\
\hline Uso de cateter venoso central $(n=145)$ & 8 & 5,5 \\
\hline Fisioterapia $(n=144)$ & 57 & 39,6 \\
\hline Cirurgias $(n=143)$ & 29 & 20,3 \\
\hline Uso de opioides $(n=119)$ & 35 & 29,4 \\
\hline Uso de hipnóticos/sedativos $(\mathrm{n}=119)$ & 59 & 49,6 \\
\hline Uso de anti-hipertensivos $(n=119)$ & 102 & 85,7 \\
\hline
\end{tabular}

* Salário mínimo - R\$957,00. 
Tabela 2. Incidência de delirium em idosos internados via emergência em um hospital terciário, conforme variáveis preditoras - Passo Fundo, RS ( $\mathrm{n}=300)$

\begin{tabular}{|c|c|c|c|c|c|}
\hline \multirow[t]{2}{*}{ Variáveis } & \multicolumn{2}{|c|}{$\begin{array}{c}\text { Com } \\
\text { delirium }\end{array}$} & \multicolumn{2}{|c|}{$\begin{array}{c}\text { Sem } \\
\text { delirium }\end{array}$} & \multirow[t]{2}{*}{$\mathbf{p}^{*}$} \\
\hline & $\mathbf{n}$ & $\%$ & $\mathbf{n}$ & $\%$ & \\
\hline Sexo & & & & & 0,332 \\
\hline Masculino & 22 & 14,1 & 134 & 85,9 & \\
\hline Feminino & 15 & 10,4 & 129 & 89,6 & \\
\hline Idade (anos completos) & & & & & 0,540 \\
\hline $60-69$ & 13 & 8,6 & 139 & 91,4 & \\
\hline $70-79$ & 12 & 13,3 & 78 & 86,7 & \\
\hline$\geq 80$ & 12 & 20,7 & 46 & 79,3 & \\
\hline Consumo de bebida alcoólica ( $n=296$ ) & & & & & 0,956 \\
\hline Sim & 5 & 11,9 & 37 & 88,1 & \\
\hline Não & 31 & 12,2 & 223 & 87,8 & \\
\hline Miniexame do Estado Mental & & & & & 0,665 \\
\hline Aprovado & 19 & 11,6 & 145 & 88,4 & \\
\hline Não aprovado & 18 & 13,2 & 118 & 86,8 & \\
\hline Número de doenças anteriores à internac & & & & & 0,025 \\
\hline $0-1$ & 4 & 4,4 & 86 & 95,6 & \\
\hline 2 & 13 & 15,5 & 71 & 84,5 & \\
\hline$\geq 3$ & 20 & 15,9 & 106 & 84,1 & \\
\hline Doença cardiovascular & & & & & 0,136 \\
\hline $\operatorname{Sim}$ & 33 & 13,8 & 207 & 86,2 & \\
\hline Não & 4 & 6,7 & 56 & 93,3 & \\
\hline Doença infecciosa & & & & & 0,101 \\
\hline $\operatorname{Sim}$ & 6 & 22,2 & 21 & 77,8 & \\
\hline Não & 31 & 11,4 & 242 & 88,6 & \\
\hline Doença psiquiátrica & & & & & 0,491 \\
\hline $\operatorname{Sim}$ & 2 & 8,0 & 23 & 92,0 & \\
\hline Não & 35 & 12,7 & 240 & 87,3 & \\
\hline Deambulação ( $n=144)$ & & & & & $<0,001$ \\
\hline Sim & 4 & 3,6 & 106 & 96,4 & \\
\hline Não & 10 & 29,4 & 24 & 70,6 & \\
\hline Uso de sonda vesical $(n=147)$ & & & & & $<0,001$ \\
\hline Sim & 8 & 30,8 & 18 & 69,2 & \\
\hline Não & 6 & 5,0 & 115 & 95,0 & \\
\hline Uso de sonda nasogástrica ( $n=146)$ & & & & & 0,385 \\
\hline $\operatorname{Sim}$ & 2 & 16,7 & 10 & 83,3 & \\
\hline Não & 12 & 9,0 & 122 & 91,0 & \\
\hline Uso de cateter venoso central $(n=145)$ & & & & & 0,779 \\
\hline Sim & 1 & 12,5 & 7 & 87,5 & \\
\hline Não & 13 & 9,5 & 124 & 90,5 & \\
\hline Fisioterapia $(n=144)$ & & & & & 0,755 \\
\hline $\operatorname{Sim}$ & 5 & 8,8 & 52 & 91,2 & \\
\hline Não & 9 & 10,3 & 78 & 89,7 & \\
\hline Cirurgias $(n=143)$ & & & & & 0,087 \\
\hline $\operatorname{Sim}$ & 5 & 17,2 & 24 & 82,8 & \\
\hline Não & 8 & 7,0 & 106 & 93,0 & \\
\hline Uso de opioides $(n=119)$ & & & & & 0,160 \\
\hline $\operatorname{Sim}$ & 6 & 17,1 & 29 & 82,9 & \\
\hline Não & 7 & 8,3 & 77 & 91,7 & \\
\hline Uso de hipnóticos/sedativos $(n=119)$ & & & & & 0,133 \\
\hline $\operatorname{Sim}$ & 13 & 10,9 & 106 & 89,1 & \\
\hline Não & 4 & 6,7 & 56 & 93,3 & \\
\hline Uso de anti-hipertensivos ( $n=119$ ) & & & & & 0,472 \\
\hline $\operatorname{Sim}$ & 12 & 11,8 & 90 & 88,2 & \\
\hline Não & 1 & 5,9 & 16 & 94,1 & \\
\hline
\end{tabular}

\section{DISCUSSÃO}

Pesquisas brasileiras recentes que avaliem a incidência de delirium em idosos internados em hospitais terciários são escassas, sobretudo se relacionadas ao setor de emergência hospitalar. Esta foi realizada em um hospital de grande porte de um município localizado na região sul do Brasil, evidenciando uma incidência de 12\% (IC95\% 9-16) em até cinco dias de internação de pacientes com idade igual ou superior a 60 anos. O resultado é semelhante ao encontrado em uma coorte histórica canadense constituída por 200 pacientes acima de 65 anos que permaneceram por ao menos 12 horas no setor de emergências, onde se constatou uma incidência de $18 \%$ de estados confusionais agudos ${ }^{11}$.

Estudo observacional prospectivo desenvolvido em um hospital universitário de Boston, Massachusetts (EUA), englobou 700 pacientes, com 65 ou mais anos de idade, que se apresentaram para cuidados no setor de emergências médicas. O resultado foi um total de $9 \%$ dos participantes delirando no momento da entrevista ${ }^{12}$, valor levemente abaixo dos encontrados em outras pesquisas, com variação entre 14\% e 24\% ${ }^{13-15}$, mas muito próximo ao ora apresentado. Incidências mais elevadas foram mostradas recentemente em associação a malignidades oncológicas, em unidades de cuidado prolongado ou em pós-operatório de cirurgias complexas, afetando de $20 \%$ a $40 \%$ dos indivíduos ${ }^{16-19}$.

$\mathrm{Na}$ amostra estudada, observou-se maior frequência de delirium em idosos restritos ao leito durante internação ( $p<$ 0,001 ). Essa restrição, não só relacionada ao espaço de confinamento comumente visto nos setores de emergência, mas também a condições clínicas do paciente que comprometem sua deambulação, está diretamente associada ao quadro. Grandes estudos, como o Delirium Day ${ }^{13}$ desenvolvido com mais de 1.800 idosos internados em hospitais e centros de reabilitação da Itália, já demonstraram que a restrição física é um forte fator precipitante para o surgimento e para a persistência de estados confusionais agudos, resultados concordantes com os encontrados e nos demais postulados na literatura 2,20 .

Também de modo semelhante à literatura ${ }^{13}$, foi observada maior incidência de delirium em idosos que fizeram uso de sonda vesical $(p<0,001)$.

Verificou-se ainda diferença de frequência do delirium de acordo com o número de doenças anteriores à internação $(p=0,025)$. Estudos com análise semelhante são escassos na literatura, e a coorte canadense não encontrou significância estatística na relação entre as duas variáveis ${ }^{11}$. A dessemeIhança, entre outros fatores, pode estar relacionada às características regionais das populações, às diferentes abordagens terapêuticas nos hospitais e às metodologias divergentes dos estudos.

Embora a maioria das pesquisas aponte doenças infecciosas, psiquiátricas, neurológicas, cardiovasculares e 
metabólicas e traumas relacionados ao surgimento do delirium ${ }^{14,17-21}$, o mesmo não foi observado neste estudo. Além disso, não se observou diferença quanto ao uso de medicamentos, ainda que esteja estabelecido na literatura que o uso de benzodiazepínicos, opioides, hipnóticos e sedativos, anti-histamínicos (especialmente os de primeira geração), anticolinérgicos, anticonvulsivantes, antidepressivos, antiparkinsonianos, antipsicóticos e barbitúricos é um forte fator de risco para o desenvolvimento do quadro, exigindo cautela na sua utilização em pacientes idosos expostos a outros fatores de risco $13,14,17,18,21,22$.

Em relação ao sexo, observou-se que 14,1\% dos homens e 10,4\% das mulheres desenvolveram delirium. Embora sugestivo, e mesmo que a literatura mostre discreta associação do distúrbio com o sexo masculino ${ }^{11,16,17}$, nenhum estudo recente conseguiu comprovar a associação entre as variáveis. Quanto à idade, entretanto, diversas pesquisas mostraram um acréscimo no risco de desenvolvimento do quadro com o avanço dela ${ }^{11,13,14,18}$.

Ainda, não foi observada relação entre o desenvolvimento do delirium e as demais variáveis abordadas (consumo de bebida alcoólica, uso de sonda nasogástrica ou cateter venoso central, realização de fisioterapia e cirurgias durante a internação), mesmo que a literatura aponte sua presença como fator precipitante do delirium ${ }^{13,22}$. Os achados divergentes podem, possivelmente, ser resultado dos cuidados aprimorados no hospital de execução da pesquisa, como também das características regionais da população.

O presente estudo retrata a realidade de um único serviço de emergência, refletindo condições particulares clínicas e demográficas que contribuem para gerar resultados específicos, não permitindo a generalização, traduzindo-se, portanto, na sua principal limitação.

Entretanto, embora distintas pesquisas brasileiras apontem dados sobre a prevalência de delirium em idosos hospitalizados ${ }^{14,23-26}$, este estudo contribui de forma importante, na medida em que traz dados recentes, nacionais, de incidência e referentes a pacientes internados via emergência, tratados clínica ou cirurgicamente, não se restringindo a pacientes de UTI, como outros encontrados na literatura ${ }^{21,27}$.

\section{CONCLUSÃO}

Este estudo retratou a incidência de delirium em pacientes com até cinco dias de internação em unidade de emergência de um hospital terciário do norte do Rio Grande do Sul, além de outros elementos relacionados a sua epidemiologia e etiologia, e os resultados encontrados, em sua maioria, vão ao encontro do que é descrito na literatura. Em virtude da relevância do tema, a pesquisa visa contribuir com a literatura nacional, levando em consideração a escassez de publicações acerca do assunto.
Diante disso, a identificação desse transtorno é fundamental na rotina de emergência dos hospitais, onde são atendidos pacientes com múltiplas doenças crônicas, pois o delirium é comumente subdiagnosticado, levando a um aumento nas taxas de morbimortalidade. Por fim, o reconhecimento do distúrbio, um ato puramente clínico, baseado na observação do paciente e na valorização dos dados fornecidos pela família, deve ser realizado sistematicamente em pacientes nesse contexto, de modo a aumentar o diagnóstico de um quadro mórbido com importantes implicações prognósticas.

\section{CONTRIBUIÇÕES INDIVIDUAIS}

Lucas Henrique Rosso - Contribuiu na concepção e desenho do estudo, na coleta, análise e interpretação dos dados e na elaboração do artigo e aprovou a versão final a ser publicada.

Guilherme Assoni Gomes - Contribuiu na coleta, análise e interpretação dos dados e na elaboração do artigo e aprovou a versão final a ser publicada.

Luis Felipe Chaga Maronezi - Contribuiu na coleta, análise e interpretação dos dados e na elaboração do artigo e aprovou a versão final a ser publicada.

Ivana Loraine Lindemann - Contribuiu na concepção e desenho do estudo, na análise e interpretação dos dados e na revisão crítica do conteúdo intelectual do artigo e aprovou a versão final a ser publicada.

Rogério Tomasi Riffel - Contribuiu na concepção e desenho do estudo e na revisão crítica do conteúdo intelectual do artigo e aprovou a versão final a ser publicada.

Júlio Cesar Stobbe - Contribuiu na concepção e desenho do estudo, na interpretação dos dados e na revisão crítica do conteúdo intelectual do artigo e aprovou a versão final a ser publicada.

\section{CONFLITO DE INTERESSES}

Todos os autores declararam não apresentar conflito de interesses.

\section{AGRADECIMENTOS}

Citando a supervisora Cristiane Roveda, os autores agradecem a toda a equipe do setor de emergência do Hospital São Vicente de Paulo de Passo Fundo, que foi fundamental para a realização do estudo.

\section{REFERÊNCIAS}

1. Francis Jr JJ, Young GB. Diagnosis of delirium and confusional states. UpToDate. 2019. 
2. Marcantonio ER. Delirium in hospitalized older adults. N Engl J Med. 2017;12(377):1456-66.

3. Dyer $(B$, Ashton $C M$, Teasdale TA. Postoperative delirium. A review of 80 primary datacollection studies. Arch Intern Med. 1995;155(5):461-5.

4. Van Rompaey B, Elseviers MM, Van Drom W, Fromont V, Jorens PG. The effect of earplugs during the night on the onset of delirium and sleep perception: a randomized controlled trial in intensive care patients. Crit Care. 2012;16(3):R73.

5. Leslie DL, Marcantonio ER, Zhang Y, Leo-Summers L, Inouye SK. One-year health care costs associated with delirium in the elderly population. Arch Intern Med. 2008;168(1):27-32.

6. Fabbri RMA, Prado LKP, Zung SP. Interconsultas psiquiátricas em pacientes idosos em hospital geral. XIV Congresso Brasileiro de Geriatria e Gerontologia. 2004.

7. American Psychiatric Association DSM-5 Task Force. Diagnostic and statistical manual of mental disorders, 5th ed.: DSM-5. Washington, DC: American Psychiatric Association; 2013.

8. Santos FS, Fraguas JR, Forlenza OV. Delirium. In: Miguel EC, Gentil V, Gattaz WF, eds. Clínica Psiquiátrica. Barueri: Manole; 2011.

9. Ringdal GI, Ringdal K, Juliebø V, Wyller TB, Hjermstad MJ, Loge JH. Using the Mini-Mental State Examination to screen for delirium in elderly patients with hip fracture. Dement Geriatr Cogn Disord. 2011:32(6):394-400.

10. Inouye SK, van Dyck CH, Alessi CA, Balkin S, Siegal AP, Horwitz RI. Clarifying confusion: the confusion assessment method. A new method for detection of delirium. Ann Intern Med. 1990;113(12):941-8.

11. Émond M, Grenier D, Morin J, Eagles D, Boucher V, Sage NL, et al. Emergency department stay associated delirium in older patients. Can Geriatr J. 2017;20(1):10-4.

12. Kennedy M, Enander RA, Tadiri SP, Wolfe RE, Shapiro NI, Marcantonio ER. Delirium risk prediction, health care utilization and mortality of elderly emergency department patients. J Am Geriatr Soc. 2014;62(3):462-9.

13. Bellelli G, Morandi A, Di Santo SG, Mazzone A, Cherubini A, Mossello E, et al. "Delirium Day": a nationwide point prevalence study of delirium in older hospitalized patients using an easy standardized diagnostic tool. BMC Med. 2016;14:106.

14. Pereira FB, Lopes MA. Delirium in elderly inpatients admitted to clinical wards: prevalence and investigation of clinical conditions in a Brazilian sample. Dement Neuropsychol. 2018;12(2):152-6.
15. American Psychiatric Association. Diagnostic and Statistical Manual of Mental Disorders. 5 a ed. Porto Alegre: Artmed; 2013. [Transtornos neurocognitivos - p. 591]

16. Sánchez-Hurtado LA, Hernández-Sánchez N, Del Moral-Armengol M, Guevara-García H, García-Guillén FJ, Herrera-Gómez A, et al. Incidence of Delirium in Critically III Cancer Patients. Pain Res Manag. 2018;2018:4193275.

17. Park SA, Tomimaru Y, Shibata A, Miyagawa S, Noguchi K, Dono K. Incidence and risk factors for postoperative delirium in patients after hepatectomy. World J Surg. 2017;41(11):2847-53.

18. Miu DKY, Chan CW, Kok C. Delirium among elderly patients admitted to a post-acute care facility and 3-months outcome. Geriatr Gerontol Int. 2016;16(5):586-92.

19. Aitken SJ, Blyth FM, Naganathan V. Incidence, prognostic factors and impact of postoperative delirium after major vascular surgery: a meta-analysis and systematic review. Vasc Med. 2017;22(5):387-97.

20. Hshieh TT, Yue J, Oh E, Puelle M, Dowal S, Travison T, et al. Effectiveness of multicomponent nonpharmacological delirium interventions. JAMA Int Med. 2015;175(4):512-20.

21. Kanova M, Kula PS, Kula R, Burda M, Janoutova J. Incidence and risk factors for delirium development in ICU patients - a prospective observational study. Biomed Paper. 2017;161(2):187-96

22. Inouye SK, Westendorp RGJ, Saczynski JS. Delirium in elderly people. Lancet. 2014;383(9920):911-22.

23. Thomazi R, Silveira LVA, Boas PJFV, Jacinto AF. Frequency of dementia among elderly admitted to a Geriatrics Inpatients Sector of a Brazilian public hospital. Dement Neuropsychol. 2018;12(1):35-9.

24. Luna $A A$, Entringer $A P$, da Silva $R C L$. Prevalência do subdiagnóstico de delirium entre pacientes internados em unidade de terapia intensiva. Rev Enferm UERJ. 2016;24(1):e6238.

25. Ohl ICB, Chavaglia SRR, OhI RIB, Lopes MCBT, Campanharo CRV, Okuno MFP, et al. Avaliação do delirium em idosos atendidos em um serviço hospitalar de emergência. Rev Bras Enferm. 2019;72(Suppl 2):153-60.

26. Martins JB, dos Santos AA, de Macedo Júnior, LJJ, Eberle CC. Avaliação da prevalência de delirium em uma unidade de terapia intensiva pública. Enferm Foco. 2019;10(3):76-81.

27. Mori S, Takeda, JRT, Carrara FSA, Cohrs CR, Zanei SSV, Whitaker IY. Incidência e fatores relacionados ao delirium em Unidade de Terapia Intensiva. Rev Esc Enferm USP. 2019:50(4):587-93 